\title{
Avaliação termográfica do efeito do piroxicam como tratamento adjuvante em neoplasias mamárias de cadelas
}

Thermographic evaluation of piroxicam's effect as an adjuvant treatment in canine mammary tumors

Evaluación termográfica del efecto del piroxicam como tratamiento adyuvante en tumores mamarios de perras

Julia Rodrigues Greghi

ORCID: https://orcid.org/0000-0002-9596-2426 Universidade Estadual de Londrina, Brasil E-mail: julia.rodrigues.greghi@uel.br

Maíra Planzo Fernandes ORCID: https://orcid.org/0000-0002-2449-6990 Universidade Estadual de Londrina, Brasil E-mail: maira.planzo.fernandes@uel.br

Aline Groth

ORCID: https://orcid.org/0000-0002-3177-6851 Universidade Estadual de Londrina, Brasil E-mail: aline_groth@hotmail.com

Letícia Amanda dos Santos Silva ORCID: https://orcid.org/0000-0002-7124-8391 Universidade Estadual de Londrina, Brasil E-mail: leticia.amanda@uel.br

Josiana de Fátima Schnitzer

ORCID: https://orcid.org/0000-0003-1805-7235 Universidade Estadual de Londrina, Brasil E-mail: josischnitzer@hotmail.com

Giovana Wingeter Di Santis

ORCID: https://orcid.org/0000-0002-9688-7181 Universidade Estadual de Londrina, Brasil E-mail: giovanaws@uel.br

Maria Isabel Mello Martins

ORCID: https://orcid.org/0000-0001-8416-2450

Universidade Estadual de Londrina, Brasil E-mail: imartins@uel.br

\begin{abstract}
Resumo
As neoplasias mamárias estão em segundo no ranking das neoplasias mais diagnosticadas em cães. As fêmeas caninas são amplamente acometidas por esta doença que, na maioria dos casos, apresenta-se na forma maligna. Sabe-se que diversos fatores estão envolvidos no desenvolvimento e determinação da malignidade destas lesões, incluindo a ação da ciclo-oxigenase-2. A utilização de anti-inflamatórios não esteroidais atuantes como inibidores desta enzima é estudada como um promissor tratamento adjuvante das neoplasias. Este estudo teve como objetivo avaliar o efeito do piroxicam na diminuição da inflamação causada pela progressão dos tumores mamários em 25 cadelas, a partir da aferição da temperatura peritumoral utilizando o recurso da termografia, durante 10 dias de administração do fármaco. Os resultados indicaram diferença significativa $(\mathrm{p}=0,003)$ entre as temperaturas médias do primeiro e último dia de tratamento. Ao realizar a avaliação das neoplasias malignas e benignas separadamente, foi possível observar que, estatisticamente, as neoplasias malignas $(\mathrm{n}=19)$ apresentaram redução significativa das temperaturas peritumorais $(\mathrm{P}=<0,001)$, contudo, as neoplasias benignas $(\mathrm{n}=6)$ não obtiveram diferença significativa da temperatura após $\mathrm{o}$ protocolo terapêutico $(\mathrm{P}=0,954)$. Dessa forma, foi possível demonstrar a eficácia da medicação em reduzir o calor causado pela inflamação e permitir avanços na elaboração de protolocos terapêuticos destinados às neoplasias mamárias em cadelas, como a inclusão do fármaco no período que antecede a realização das cirurgias de mastectomia. Palavras-chave: Canino; Oncologia; Glândulas mamárias; Inflamação; Inibidores COX-2.
\end{abstract}

\section{Abstract}

Mammary tumors are the second most diagnosed neoplasms in dogs. Canine females are largely affected by malignant mammary tumours. Several factors are involved in the development and determination of the malignancy of these 
lesions, including the action of cyclooxygenase-2. Use of non-steroidal anti-inflammatory drugs as enzyme-inhibiting drugs has been studied as a promising adjuvant treatment for neoplasms. The aim of this study was to evaluate the effect of piroxicam in reducing inflammation caused by mammary tumours in 25 bitches during 10 days of administration based on the measurement of peritumoral temperature using thermography. The results indicated a significant difference $(\mathrm{p}=0,003)$ between the average temperatures at the first and last day of treatment. When performing the evaluation of malignant and benign neoplasms separately, it was possible to observe that, statistically, malignant neoplasms $(\mathrm{n}=19)$ showed a significant reduction in peritumoral temperatures $(\mathrm{P}=<0.001)$, however, benign neoplasms $(n=6)$ have not obtained a significant difference in temperature after the therapeutic protocol $(\mathrm{P}=0.954)$. Therefore, it was possible to demonstrate the efficacy of the medication in reducing the heat caused by inflammation and allowing advances in the development of therapeutic protocols for mammary tumors in bitches, such as the inclusion of the drug in the period prior to the mastectomy surgeries.

Keywords: Canine; Oncology; Mammary gland; Inflammation; COX-2 Inhibitors.

\section{Resumen}

Los tumores mamarios ocupan el segundo lugar em el ranking de los cánceres más frecuentemente diagnosticados en perros. Las perras son las más afectadas por esta enfermedad que en la mayoría de los casos se presenta de forma maligna. Se sabe que varios factores están involucrados en el desarrollo y determinación de la malignidad de estas lesiones, incluida la acción de la ciclooxigenasa-2. Se estudia la utilización de antiinflamatorios no esteroides que actúan como inhibidores enzimáticos como un tratamiento adyuvante prometedor para neoplasias. Este estudio tuvo como objetivo evaluar el efecto del piroxicam en la reducción de la inflamación causada por la progresión de los tumores de mama en 25 perras, a partir de la medición de la temperatura peritumoral, mediante termografía, durante 10 días de administración del fármaco. Los resultados indicaron una diferencia significativa $(\mathrm{p}=0,003)$ entre las temperaturas medias del primer y último día de tratamiento. Al realizar la evaluación de neoplasias malignas y benignas por separado, se pudo observar que, estadísticamente, neoplasias malignas $(n=19)$ mostraron una reducción significativa de las temperaturas peritumorales $(\mathrm{P}=<0,001)$, sin embargo, neoplasias benignas $(\mathrm{n}=6)$ no obtuvieron una diferencia significativa de temperatura después del protocolo terapéutico $(\mathrm{P}=0,954)$. Así, se pudo demostrar la eficacia de la medicación en la reducción del calor provocado por la inflamación y permitiendo avances en el desarrollo de protocolos terapéuticos para los tumores mamarios, como la inclusión del fármaco en el período previo a la realización de la mastectomía.

Palabras clave: Perros; Oncología; Glándula mamaria; Inflamación; Inhibidores COX-2.

\section{Introdução}

As neoplasias mamárias estão em segundo no ranking de neoplasias mais diagnosticadas em cães. Ocorrem com maior frequência nas fêmeas e aproximadamente 68\% apresentam-se de forma maligna (Henry, 2012; Tedardi, Kimura, Mendonça, \& Dagli, 2016). Atualmente, existem diversos estudos em busca de marcadores biológicos para auxílio no diagnóstico, melhorias na terapêutica e definição de prognóstico, no que diz respeito ao desenvolvimento e agressividade tumoral (Szweda, Rychlik, Babinska, \& Pomianowski, 2020). Apesar da importância dos fatores hormonais, dietéticos e idade no desenvolvimento das neoplasias mamárias, sabe-se que diversos outros mecanismos estão envolvidos na oncogênese, incluindo um importante biomarcador que é a enzima ciclo-oxigenase-2 (De Nardi, Ferreira, \& Assunção, 2016).

A ciclo-oxigenase-2 (COX-2) é uma enzima induzida em casos de estímulos específicos como processos inflamatórios, quadros de hipóxia e ativação de oncogenes (Doré, 2011). Acredita-se que a expressão exacerbada da enzima nos casos de inflamações crônicas possa acarretar o surgimento de neoplasias, como visto por Liu et al. (2001) ao induzir a super expressão da COX-2 em ratos e observar a ocorrência de hiperplasia, displasia e tumores mamários. Isso se deve por atuarem no aumento da divisão celular, na angiogênese, invasão e metástase tumoral e supressão da resposta imunológica antitumoral (Heller et al., 2005).

Partindo desse princípio, a utilização de fármacos anti-inflamatórios não esteroidais (AINEs) surgem como um promissor tratamento adjuvante de neoplasias, uma vez que atuam inibindo a enzima COX-2 (Rodaski, \& De Nardi, 2008). Existem protocolos terapêuticos que envolvem o uso de AINEs como o carprofeno, firocoxibe e o piroxicam para o tratamento das lesões (De Nardi et al., 2016; Cassali et al., 2019).

Para acompanhamento e mensuração do processo inflamatório, existem alguns métodos descritos na literatura, como a dosagem de proteínas de fase aguda, avaliação histopatológica dos componentes teciduais e, até mesmo, análise 
imunohistoquímica para detecção dos marcadores pró-inflamatórios (Carvalho et al., 2016; Rang, Ritter, Flower, \& Henderson, 2016a). Com o auxílio da termografia é possível verificar a progressão ou regressão do processo inflamatório de forma não invasiva, a partir da mensuração da temperatura do local onde o tumor está instalado (Roberto \& Souza, 2014), visto que este evento promove angiogênese e vasodilatação, aumentando a temperatura local (Rang et al., 2016a). Este método também é utilizado para detecção precoce de câncer de mama em mulheres levando em consideração a diferença de temperatura em relação à mama não acometida (Carvalho et al., 2016).

O objetivo deste estudo foi avaliar, pela utilização da termografia, a dinâmica da inflamação nas neoplasias mamárias em cadelas antes e após o tratamento com piroxicam e estimar a eficiência do fármaco como forma de tratamento adjuvante.

\section{Metodologia}

Este estudo foi realizado de acordo com o Comitê de Ética em Experimentação Animal da Universidade Estadual de Londrina com base na Lei Federal 11.794, de 8 de outubro de 2008 aprovado sob o número 5609.2017.46.

Foram incluídas no estudo 25 cadelas com neoformações em mama, de forma aleatória, independentemente do número de lesões, peso ou idade. No primeiro atendimento (D0), foi realizado exame clínico geral da paciente, mensuração do tamanho dos nódulos, classificação das características macroscópicas e citologia aspirativa por agulha fina dos nódulos da(s) cadeia(s) mamária(s), além da imagem termográfica das cadeias mamárias. No caso de múltiplas lesões, a mensuração da temperatura foi realizada ao redor da neoformação de maior tamanho nos dois momentos. O tratamento prescrito foi o piroxicam de forma manipulada na dose de $0,3 \mathrm{mg} / \mathrm{kg}$ por via oral uma vez ao dia durante 10 dias. Após 10 dias (D10), os animais retornaram para avaliação da evolução do quadro e repetição da termografia.

Nos dois momentos, os animais foram mantidos em local fresco, tricotomizados na região abdominal e posicionados em decúbito dorsal a uma distância de, aproximadamente, um metro da câmera, com o intuito de aferir a temperatura de ambas as cadeias mamárias para padronização. O termógrafo utilizado foi o Flir ${ }^{\circledR}$, modelo T440, Boston, USA e a reprodução e leitura das imagens feita pelo software Flir QuickReport. Todos os animais foram submetidos ao estadiamento tumoral, seguindo o sistema TNM (Cassali et al., 2019), para posterior realização do procedimento cirúrgico de mastectomia total unilateral da cadeia mamária acometida juntamente com exérese dos linfonodos regionais. As amostras foram enviadas para realização de exame histopatológico, portanto, foram mantidas em formaldeído $10 \%$ por 48 horas, submetidas ao processamento histológico, embebidas em parafina e coradas conforme o protocolo de hematoxilina e eosina.

Os resultados foram tabulados em Microsoft Excel® e a análise estatística foi processada no programa SigmaPlot pelo teste t pareado, com nível de confiança de $95 \%$ ( $\mathrm{p}<0,05)$.

\section{Resultados e Discussão}

No presente trabalho, a mensuração e comparação das temperaturas peritumorais nos dois momentos permitiu a observação da dinâmica inflamatória local, constatando uma diminuição significativa das médias antes $\left(\mathrm{D} 0=37,41^{\circ} \mathrm{C}\right)$ e após $\left(\mathrm{D} 10=36,91^{\circ} \mathrm{C}\right)$ a administração do fármaco $(\mathrm{p}=0,003)$. Observou-se que 18 dos 25 animais $(72 \%)$ apresentaram redução significativa da temperatura após tratamento. O menor nódulo avaliado possuía 0,5 centímetros de diâmetro, enquanto o maior apresentava $13 \times 5 \times 2,5$ centímetros, os quais apresentaram redução na temperatura peritumoral de $1{ }^{\circ} \mathrm{C}$ e $0,8^{\circ} \mathrm{C}$ após o tratamento, respectivamente. Os dados obtidos na avaliação da temperatura peritumoral no primeiro e último dia de tratamento, a localização dos tumores e estadiamento dos nódulos estão descritos na Tabela 1.

A idade média das cadelas avaliadas foi de 10 anos \pm 2 , semelhante ao que é descrito na literatura que indica a incidência das neoplasias mamárias em animais com faixa etária entre sete e 12 anos (De Nardi et al., 2016). Em relação a 
predisposição racial, embora seja reportado maior incidência de tumores nas raças Poodle, Dachshund, Yorkshire Terrier, Cocker Spaniel, Pastor Alemão, Boxer e Fox Terrier (De Nardi et al., 2016), este estudo encontrou predominância de $68 \%$ em cadelas sem raça definida.

A avaliação da temperatura das glândulas mamárias em estudos recentes indica que há aumento significativo nos tecidos afetados quando comparados a tecidos mamários saudáveis, independentemente do tamanho do tumor (Pavelski et al., 2015). Nas neoplasias mais agressivas, de crescimento rápido, maior tamanho, ulceradas e aderidas, a presença da COX-2 pode ser mais elevada, além da alta expressão de fatores de crescimento vascular e tumoral, promovendo maior aumento da temperatura (Anadol et al., 2017; Queiroga, Pires, Parente, Gregório, \& Lopes, 2011). Sendo assim, foi possível constatar que a administração do fármaco pode ser recomendada em diferentes estádios de desenvolvimento tumoral e para diferentes tipos tumorais.

A partir dos resultados dos exames histopatológicos, após a mastectomia, foi observada prevalência dos carcinomas em tumor misto (14/25). Contudo, também foram diagnosticadas neoplasias correspondentes a tumor misto benigno (4/25), carcinoma papilar (2/25), carcinoma micropapilar (1/25), carcinossarcoma (1/25), adenoma ductal (1/25), hiperplasia ductal (1/25) e hemangiossarcoma (1/25). Quanto aos relatos existentes sobre os tipos tumorais mais recorrentes, é observada predominância de neoplasias epiteliais malignas em relação às benignas e mesenquimais (Cassali et al., 2019). Neste estudo foi observada prevalência de neoplasias de origem epitelial, porém com caráter misto. Entretanto, os diferentes autores divergem quanto à classificação dos tumores. Carvalho et al. (2016) encontraram predomínio dos carcinomas tubulopapilares enquanto que Pavelski et al. (2015) observaram maior número de carcinomas papilares em suas análises. As principais mamas acometidas foram as abdominais caudais e inguinais, como descrito na Tabela 1, corroborando com os dados da literatura, tal fato justifica-se pela maior quantidade de tecido mamário presente nessas regiões da cadeia (De Nardi et al., 2016; Pavelski et al., 2015). 
Tabela 1. Temperatura peritumoral dos nódulos selecionados nos dia 0 e dia 10 de tratamento com o piroxicam, localização das lesões, estadiamento tumoral e resultados dos exames histopatológicos após a realização da mastectomia.

\begin{tabular}{|c|c|c|c|c|c|c|}
\hline Animal & D0 & D10 & (D10-D0) & Mama acometida & $\begin{array}{l}\text { Estadiamento TNM } \\
\text { (Cassali et al., 2019) }\end{array}$ & Histopatológico \\
\hline 1 & 37,70 & 36,70 & 1,00 & Torácica cranial E & I & Hiperplasia ductal atípica \\
\hline 2 & 38,40 & 37,20 & 1,20 & Inguinal E & II & Carcinossarcoma \\
\hline 3 & 37,50 & 36,00 & 1,50 & Inguinal E & III & Hemangiossarcoma \\
\hline 4 & 38,50 & 36,70 & 1,80 & Abdominal caudal E & I & Carcinoma em tumor misto \\
\hline 5 & 37,50 & 36,10 & 1,40 & Torácica caudal D & II & Carcinoma em tumor misto \\
\hline 6 & 37,00 & 36,80 & 0,20 & Abdominal caudal D & I & Carcinoma em tumor misto \\
\hline 7 & 36,80 & 37,20 & $-0,40$ & Inguinal D & I & Carcinoma em tumor misto \\
\hline 8 & 35,70 & 36,50 & $-0,80$ & Abdominal caudal E & I & Tumor misto benigno \\
\hline 9 & 37,50 & 38,20 & $-0,70$ & Inguinal D & I & Adenoma ductal \\
\hline 10 & 37,60 & 37,20 & 0,40 & Inguinal D & I & Carcinoma em tumor misto \\
\hline 11 & 37,90 & 37,10 & 0,80 & Abdominal caudal E & IV & Carcinoma em tumor misto \\
\hline 12 & 37,90 & 37,60 & 0,30 & Inguinal D & III & Carcinoma em tumor misto \\
\hline 13 & 37,70 & 38,00 & $-0,30$ & Abdominal caudal D & III & Carcinoma em tumor misto \\
\hline 14 & 37,60 & 37,70 & $-0,10$ & Inguinal E & I & Tumor misto benigno \\
\hline 15 & 36,60 & 36,40 & 0,20 & Inguinal D & II & Carcinoma em tumor misto \\
\hline 16 & 37,10 & 36,70 & 0,40 & Inguinal D & II & Carcinoma em tumor misto \\
\hline 17 & 37,50 & 36,60 & 0,90 & Inguinal D & III & Carcinoma em tumor misto \\
\hline 18 & 37,20 & 35,40 & 1,80 & Abdominal caudal E & II & Carcinoma papilar \\
\hline 19 & 35,50 & 35,10 & 0,40 & Inguinal E & I & Tumor misto benigno \\
\hline 20 & 37,80 & 36,90 & 0,90 & Abdominal caudal D & II & Carcinoma em tumor misto \\
\hline 21 & 37,30 & 37,60 & $-0,30$ & Abdominal caudal E & III & Carcinoma micropapilar \\
\hline 22 & 38,50 & 37,90 & 0,60 & Abdominal cranial D & II & Carcinoma em tumor misto \\
\hline 23 & 37,30 & 37,20 & 0,10 & Abdominal caudal D & I & Tumor misto benigno \\
\hline 24 & 36,60 & 36,90 & $-0,30$ & Inguinal D & II & Carcinoma em tumor misto \\
\hline 25 & 38,60 & 36,90 & 1,70 & Inguinal E & III & Carcinoma papilar \\
\hline Média & 37,41 & 36,90 & & & & \\
\hline $\mathrm{DP}$ & 0,75 & 0,77 & & & & \\
\hline
\end{tabular}

Nota . D0 = Primeiro dia do tratamento; D10 = Após 10 dias de tratamento; D10-D0 = Diferença das temperaturas peritumorais do primeiro e último dia de tratamento; D = Direita; E = Esquerda; DP = Desvio padrão. Fonte: Autores.

Ao realizar a avaliação da temperatura peritumoral dos nódulos de classificação histopatológica maligna e benigna separadamente, foi possível observar que, estatisticamente, as neoplasias malignas $(\mathrm{n}=19)$ apresentaram redução significativa das temperaturas peritumorais $(\mathrm{P}=<0,001)$ enquanto que as neoplasias benignas $(\mathrm{n}=6)$ não obtiveram diferença significativa da temperatura após o protocolo terapêutico $(\mathrm{P}=0,954)$. Estudo realizado por Salvador et al. (2013) evidenciou aumento na expressão de COX-2 conforme maior o grau de malignidade e agressividade dos tumores, tal fato nos permite compreender a obtenção de resultados positivos quando utilizado o tratamento adjuvante em neoplasias mamárias de caráter maligno, como os carcinomas.

Apesar de ser um fármaco fracamente seletivo para COX-2, o piroxicam possui um valor de mercado mais acessível quando comparado aos coxibes (inibidores seletivos COX-2), além de possuir mínimos efeitos gastrointestinais quando 
comparado à administração dos demais fármacos da mesma classe (Rang, Ritter, Flower, \& Henderson, 2016b). Além disso, a literatura traz estudos favoráveis quanto à sobrevida de animais tratados com o piroxicam em associação à quimioterapia convencional quando comparados com aqueles tratados com protocolos tradicionais de quimioterapia nos casos de carcinoma mamário inflamatório (Souza, Toledo-Piza, Amorin, Barboza, \& Tobias, 2009). Essas constatações e os resultados obtidos neste trabalho sugerem a capacidade do piroxicam de reduzir os níveis de substâncias pró-inflamatórias produzidas pela enzima COX-2 que promovem o aumento da temperatura peritumoral.

Além da visualização em imagem do efeito anti-inflamatório do fármaco, houve relatos favoráveis dos tutores em relação ao estado geral dos animais, corroborando com a literatura que sugere o piroxicam como tratamento de escolha para dores crônicas (Rang et al., 2016b). Durante a anamnese, os proprietários foram questionados quanto à evolução do estado geral dos animais após a administração do piroxicam, 20 dos 25 animais do estudo apresentaram melhora no quadro e no bemestar, foi relatada a melhora do apetite, diminuição da dor e, em alguns casos, redução da secreção presente nas glândulas mamárias, sinais que ratificam o efeito do fármaco tanto no controle da inflamação quanto da dor oncológica.

Os resultados favoráveis apresentados por este experimento permitem que trabalhos futuros possam ser realizados a fim de associar a avaliação do processo inflamatório com a termografia aos métodos diagnósticos, como a imunohistoquímica, para quantificação da expressão da enzima em cada um dos tipos tumorais e abrir caminhos para estudos com diferentes fármacos.

\section{Conclusão}

Baseado nos resultados obtidos é possível incluir o piroxicam como opção de fármaco adjuvante no início do tratamento de neoplasias mamárias malignas em cadelas e a termografia como um método eficiente de acompanhamento da dinâmica inflamatória.

\section{Referências}

Anadol, E., Saglam, A. S. Y., Gultiken, N., Karakas, K., Alcigir, E., Alkan, H., \& Kanca, H. (2017). Expression of iNOS, COX-2 and VEGF in canine mammary tumours and non-neoplastic mammary glands: association with clinicopatholgical features and tumour grade. Acta Veterinaria Hungarica, 65(3), 382-393.

Cassali, G. D., Jark, P. C., Gamba, C., Damasceno, K. A., Estrela-Lima, A., De Nardi, A. B., Ferrerira, E., Horta, R. S., Firmo, B. F., Sueiro, F. A. R., Rodrigues, L. C. S., \& Nakagaki, K. Y. R. (2019). Consensus regarding the diagnosis, prognosis and treatment of canine and feline mammary tumors. Brazilian Journal of Veterinary Pathology, 13(3), 555-574.

Carvalho, M. I., Pires, I., Prada, J., Raposo, T. P., Gregório, H., Lobo, L., \& Queiroga, F. L. (2016). High COX-2 expression is associated with increased angiogenesis, proliferation and tumoural inflammatory infiltrate in canine malignantmammary tumours: a multivariate survival study. Veterinary and Comparative Oncology, 15(2),619-631.

De Nardi, A. B., Ferreira, T. M. M. R., \& Assunção, K. A. (2016). Neoplasias Mamárias. In A. B. De Nardi \& C. R. Daleck. Oncologia em cães e gatos (pp. 499-515). Roca.

Doré, M. (2011). Cyclooxygenase-2 expression in animal cancers. Veterinary Pathology, 48(1), 254-265.

Heller, D. A., Clifford, C. A., Goldschmidt, M. H., Holt, D. E., Shofer, F. S., Smith, A. \& Sorenmo K. (2005). Cyclooxygenase-2 expression in associated with histologic tumor type in canine mammary carcinoma. Veterinary Pathology, 42(6), 776-780.

Henry, C. J. (2012). The Etiology of Cancer - Section B: Chemical, Physical, and Hormonal Factors. In S. J. Withrow, D. M. Vail, \& R. L. Page. Withrow \& MacEwen`s Small Animal Clinical Oncology (pp. 1-29). Saunders.

Liu, C. H., Chang, S. H., Narko, K., Trifan, O. C., Wu, M. T., Smith, E., Haudenschild, C., Lane, T. F., \& Hla, T.(2001). Overexpression of cyclooxygenase-2 is sufficient to induce tumorigenesis in transgenic mice. Journal of Biological Chemestry, 276(21), 18563-18569.

Pavelski, M., Silva, D. M., Leite, N. C., Junior, D. A., De Sousa, R. S., Guerios, S. D., \& Dornbusch, P. T. (2015). Infrared Thermography in Dogs with Mammary Tumors and Healthy Dogs. Journal of Veterinary Internal Medicine, 29(6), 1578-1583.

Queiroga, F. L., Pires, I., Parente, M., Gregório, H., \& Lopes, C. (2011). COX-2 over-expression correlates with VEGF and tumour angiogenesis in canine mammary cancer. The Veterinary Journal, 189(1), 77-82. 
Research, Society and Development, v. 10, n. 2, e18710212236, 2021

(CC BY 4.0) | ISSN 2525-3409 | DOI: http://dx.doi.org/10.33448/rsd-v10i2.12236

Rang, H. P., Ritter, J. M., Flower, R. J., \& Henderson, G. (2016a). Mecanismos celulares: defesa do hospedeiro. In H. P. Rang, J. M. Ritter, R. J. Flower, \& G. Henderson. Rang \& Dale: Farmacologia (209-236). Elsevier.

Rang, H. P., Ritter, J. M., Flower, R. J., \& Henderson, G. (2016b). Fármacos anti-inflamatórios e imunossupressores. In H. P. Rang, J. M. Ritter, R. J. Flower, \& G. Henderson. Rang \& Dale: Farmacologia (754-794). Elsevier.

Roberto, J. V. B., \& Souza, B. B. (2014) Utilização da termografia de infravermelho na medicina veterinária e na produção animal. Journal of Animal Behaviour and Biometeorology, 2(3), 73-84.

Rodaski, S., \& De Nardi, A. B. (2008). Quimioterapia antineoplásica em cães e gatos. MedVet Livros.

Salvador, R. C. L., Terra, E. M., Raposo, T. M. M., Alves, C. E. F., Costa, M. .T., \& Amorim, R. L. (2013) Increased cycloxygenase-2 (COX-2) expression in associated with the progression and aggression of canine mammary tumors. Archives of Veterinary Science, 18(2), 205-207.

Souza, C. H. M., Toledo-Piza, E., Amorin, R., Barboza, A., \& Tobias, K. M. (2009). Inflammatory mammary carcinoma in 12 dogs: Clinical features, cyclooxygenase-2 expression, and response to piroxicam treatment. Canadian Veterinary Journal, 50(5), 506-510.

Tedardi, M. V., Kimura, K. C., Mendonça, P. P., \& Dagli, M. L. Z. (2016). Epidemiologia e Etiologia do Câncer. In A. B. De Nardi, \& Daleck, C. R. Oncologia em cães e gatos (pp. 1-28). Roca.

Szweda, M., Rychlik, A., Babinska, I., \& Pomianowski, A. (2020). Cyclooxygenase-2 as a biomarker with diagnostic, therapeutic, prognostic, and predictive relevance in small animal oncology. Journal of Veterinary Research, 64(1), 151-160. 